Draft VERSiOn OCTOBER 31, 2018

Preprint typeset using $\mathrm{L}^{A} \mathrm{~T}_{\mathrm{E}} \mathrm{X}$ style emulateapj v. 08/22/09

\title{
THE SDSS-UKIDSS FUNDAMENTAL PLANE OF EARLY-TYPE GALAXIES
}

\author{
La Barbera, F. ${ }^{1}$, Busarello, G. ${ }^{1}$, Merluzzi, P. ${ }^{1}$, De la Rosa, I. ${ }^{2}$, Coppola, G. ${ }^{3}$, Haines, C.P. ${ }^{4}$ \\ Draft version October 31, 2018
}

\begin{abstract}
We derive the Fundamental Plane (FP) relation for a sample of 1430 early-type galaxies in the optical ( $\mathrm{r}$ band) and the near-infrared ( $\mathrm{K}$ band), by combining SDSS and UKIDSS data. With such a large, homogeneous dataset, we are able to assess the dependence of the FP on the waveband. Our analysis indicates that the FP of luminous early-type galaxies is essentially waveband independent, with its coefficients increasing at most by $8 \%$ from the optical to the NIR. This finding fits well into a consistent picture where the tilt of the FP is not driven by stellar populations, but results from other effects, such as non-homology. In this framework, the optical and NIR FPs require more massive galaxies to be slightly more metal rich than less massive ones, and to have highly synchronized ages, with an age variation per decade in mass smaller than a few percent.
\end{abstract}

Subject headings: Galaxies: fundamental parameters - Galaxies: evolution

\section{INTRODUCTION}

Early-type galaxies (ETGs) populate a twodimensional surface in the space of parameters that reflect size (effective radius), density (mean surface brightness), and kinetic temperature (velocity dispersion) (Djorgovski 1992). A key feature of the Fundamental Plane (FP; Dressler et al. 1987; Diorgovski \& Davis 1987) is its deviation ('tilt') from the virial theorem, which may be interpreted as a variation of the $\mathrm{M} / \mathrm{L}$ ratio along the sequence of ETGs and/or the breaking of homology assumption, i.e. the fact that, for all galaxies, the observed parameters have the same power-law dependence on the corresponding physical quantities (namely, the central velocity dispersion on kinetic energy, the effective radius on 'gravitational' radius, and the effective surface brightness on the overall light profile, see e.g. Diorgovski \& Santiago 1993). Despite all the observational efforts, the origin of the tilt is still under debate. The change of the $\mathrm{M} / \mathrm{L}$ ratio can be explained by a change in either the stellar population (e.g. Prugniel \& Simien 1996) or dark matter content with galaxy mass (Ciotti et al. 1996). Both structural and dynamical non-homology have also been invoked as physical explanations of the observed tilt (see e.g. Hiorth \& Madsen 1995, Capelato et al. 1995, Graham \& Colless 1997, and Busarello et al. 1997). Recently, Trujillo et al. (2004, from now TBB04) showed that the tilt is mostly driven by dynamical and structural non-homology, while stellar populations account for only a small fraction of it. Bolton et al. (2007) argued that the tilt is more likely because of a variation of the dark matter content with mass, still favoring a picture where stellar populations play a minor role. Since the contribution of different stellar populations to galaxy luminosity is expected to be wavelength-

\footnotetext{
${ }^{1}$ INAF - Osservatorio Astronomico di Capodimonte, Napoli, Italy,

${ }^{2}$ Instituto de Astrofisica de Canarias, Tenerife, Spain,

${ }^{3}$ University of Naples Federico II, Department of Physics, Napoli, Italy,

4 School of Physics and Astronomy, University of Birmingham, Birmingham, UK
}

dependent, while other effects (e.g. non-homology) are not, the dependence of the FP on wavelength directly informs on how properties of the stellar populations change with mass, which is a crucial point to understand galaxy formation and evolution.

Previous studies of the wavelength dependence led to contradictory results. Pahre et al. (1998b) and Scodeggio et al. (1998) found the tilt to significantly decrease from optical to NIR wavelengths, interpreting this result as an increase of age and metallicity with mass. Mobasher et al. (1999) and Zibetti et al. (2002) found only a small decrease of the tilt with wavelength, with the FP still being significantly tilted in the NIR. However, Bernardi et al. (2003b), deriving the FP for ETGs observed in the Sloan Digital Sky Survey (SDSS), found evidence for the FP to be wavelength independent from the $g$ to the $z$ bands. Several different effects can produce this puzzling picture. The FP in different wavebands has often been derived for small samples, with inhomogeneous measurements of galaxy parameters, different selection criteria (e.g. galaxy samples spanning different ranges in magnitude and/or velocity dispersion), and with different fitting methods. The FP by Bernardi et al. (2003b) avoided all these problems by analyzing the same sample of galaxies at different wavebands, but it was limited to the short wavelength baseline provided by the SDSS. In the present work, for the first time, we derive the FP by using the same, large, homogeneous sample of ETGs over the wide wavelength baseline provided by the r- and K-band data of the SDSS and the UKIRT Infrared Deep Sky Survey (UKIDSS).

The layout of the paper is the following. In Sec. 2, we describe the selection of the sample, while Sec. 3 details how we obtain the r- and K-band structural parameters, and the central velocity dispersions. Sec. 4 deals with the comparison of the r- and K-band FPs. In Sec. 5, we show how the optical and NIR FPs constrain the variation of stellar population parameters along the galaxy sequence. The discussion follows in Sec.6. Throughout the paper, we adopt the cosmology $\mathrm{H}_{0}=75 \mathrm{~km} \mathrm{~s}^{-1} \mathrm{Mpc}^{-1}, \Omega_{\mathrm{m}}=0.3$, and $\Omega_{\Lambda}=0.7$.

2. SAMPLE SELECTION 
We select a sample of ETGs, with available K-band photometry from the second data release of UKIDSS, and r-band photometry and central velocity dispersions from the fifth data release (DR5) of SDSS. First, a complete volume-limited catalog of galaxies is defined, consisting of all the 105036 objects in DR5 with an r-band absolute magnitude $\mathrm{M}_{r}<-20$, and a spectroscopic redshift in the range of 0.05 to 0.095 . Absolute magnitudes are obtained from the SDSS r-band Petrosian magnitudes, $\mathrm{k}$-corrected to redshift 0.1 by using the kcorrectv4_1_4 software (Blanton et al. 2003). The lower redshift limit is chosen to minimize the aperture bias (Gómez et al. 2003), while the upper redshift limit guarantees a high level of completeness (see Sorrentino et al. 2006). ETGs are defined according to the SDSS spectroscopic parameter eclass, that classifies the spectral type on the basis of the principal component analysis technique, and the photometric parameter fracDev $v_{r}$, which measures the fraction of galaxy light that is fitted by a de Vaucouleurs law. Following Bernardi et al. (2003a), we define as ETGs those objects with eclass $<0$ and rracDev $_{r}>0.8$, resulting in a list of 47061 galaxies. Out of them, we retain only those 33628 galaxies with available central velocity dispersion, $\sigma_{0}$, between 70 and $420 \mathrm{~km} \mathrm{~s}^{-1}$. These cuts are required to obtain reliable $\sigma_{0}$ 's from the SDSSDR5 database. All the selected galaxies have spectra with median per-pixel $S / N$ larger than 10 , which is a further requirement to retrieve reliable SDSS velocity dispersions ${ }^{5}$. The SDSS catalog is cross-matched with UKIDSS, resulting in 1570 galaxies. We notice that all the SDSS galaxies with reliable velocity dispersion, that are covered by the UKIDSS survey, are then included in this sample. In other words, the matching with UKIDSS does not change the magnitude limit of the present sample. We select only those galaxies observed in K-band images with good seeing $\left(F W H M \leq 1^{\prime \prime}\right)$. This selection reduces the sample size by only $10 \%$, and excludes cases where structural parameters might be affected by large uncertainties. Since all the r-band images have FWHM smaller than $1.4^{\prime \prime}$ and the galaxies in our sample have on average effective radii larger in the optical than in the K-band (see below), we do not apply any seeing selection to the SDSS photometry. The above procedure leads to a final sample of 1430 galaxies.

\section{FP PARAMETERS}

The photometric parameters entering the FP, namely, the effective radius, $r_{\mathrm{e}}$, and the mean surface brightness within that radius, $\langle\mu\rangle_{\mathrm{e}}$, are derived using 2DPHOT (La Barbera et al. 2008). The r- and K-band images are processed by adopting the same 2DPHOT options, allowing homogeneous structural parameters to be derived between both bands. For each galaxy, a local PSF model is computed by fitting simultaneously the four closest stars to that galaxy on the image. Structural parameters, i.e. the effective parameters and the Sersic index $n$ (shape parameter), are then derived by modeling the galaxy images with two-dimensional seeing-convolved Sersic models. Effective radii are converted to physical units by using the angular diameter distance correspond-

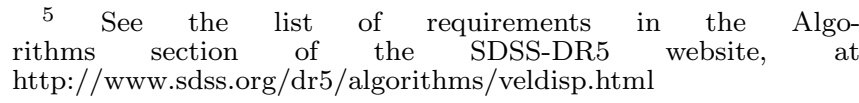

ing to the DR5 spectroscopic redshift, $z$, of each galaxy. Mean surface brightnesses are de-reddened using the extinction maps of Schlegel, Finkbeiner and Davis (1998), are corrected for cosmological dimming, by subtracting the term $7.5 \log (1+\mathrm{z})$, and are k-corrected to redshift 0.1 with the kcorrectv4_1_4 software (Blanton et al. 2003). In order to estimate the accuracy on structural parameters, we use 160, out of the 1430, galaxies having repeated observations in different UKIDSS frames. We find that the averaged differences between repeated measurements of structural parameters are fully consistent with zero, amounting to $-0.01 \pm 0.01,-0.037 \pm 0.04$, and $0.005 \pm 0.01$ for $\log r_{\mathrm{e}},\langle\mu\rangle_{\mathrm{e}}$ and $\log n$, respectively. The rms values of these differences amount to $32 \%$ in $r_{\mathrm{e}}, 0.6 \mathrm{mag} / \operatorname{arcsec}^{2}$ in $\langle\mu\rangle_{\mathrm{e}}$, and $25 \%$ in $n$. Notice that the scatter in $\log r_{\mathrm{e}}$ is fully consistent with the typical accuracy of the measurement of the half-light radii (Kelson et al. 2000). The quantity $\left.\log r_{\mathrm{e}}-0.3<\mu\right\rangle_{\mathrm{e}}$, that is the relevant photometric parameter entering the $\mathrm{FP}$, has an uncertainty of only $7 \%$, as expected due to the correlation of measurement errors of the effective parameters. The comparison of $\mathrm{r}$ - and K-band structural parameters for the present sample is fully consistent with what is found in our previous studies (e.g. La Barbera et al. 2004). In particular, the mean ratio between $\mathrm{r}$ - and K-band radii is $-0.11 \pm 0.01$ dex, i.e. on average effective radii decrease by $\sim 25 \%$ from the optical to the NIR. This value is consistent with that of $\sim 20 \%$ found by Pahre et al. (1998a), and is in agreement with the existence of negative color gradients in early-type galaxies. Sersic indices are fully consistent between optical and NIR. The average ratio of r- to K-band $n$ values amounts to $-0.007 \pm 0.009$ dex. Central velocity dispersions are retrieved from DR5 and are corrected as in Bernardi et al. (2003b) to a relative aperture of $r_{\mathrm{e}} / 8$, following Jørgensen et al. (1995). As shown by Bernardi (2007), for $\sigma_{0}<150 \mathrm{~km} \mathrm{~s}^{-1}$ the DR5 velocity dispersions are slightly overestimated. This small bias increases up to $12 \%$ at $\sigma_{0} \sim 100 \mathrm{~km} \mathrm{~s}^{-1}$. We remove this effect by applying the correction curve shown in fig. 4 of Bernardi (2007) (see the grey line in the upper-left panel $)^{6}$. The r- and K-band effective parameters, as well as the corrected DR5 velocity dispersions are given in Tab. 1 (fully available in electronic form) for all the 1430 galaxies analyzed in the present study. Columns in the table provide the following quantities. Columns 1 and 2 report right ascension (RA) and declination (DEC) in units of degrees. Columns 3 and 4 provide the logarithm of the effective radius (in unis of $k p c$ ) and the effective mean surface brightness (in unis of $\mathrm{mag} / \operatorname{arcsec}^{2}$ ) in the r-band. Columns 5 and 6 list the same quantities as columns 3 and 4 but for the K-band. Column 7 reports the corrected DR5 velocity dispersions.

\section{THE SDSS AND UKIDSS FUNDAMENTAL PLANES}

We adopt the usual representation of the FP, $\log r_{\mathrm{e}}=$ $a \log \sigma_{0}+b\langle\mu\rangle_{\mathrm{e}}+c$, where $a$ and $b$ are the "slopes", and $c$ is the offset. These coefficients were derived by minimizing the sum of the absolute residuals around the plane. This method has the advantage of being

6 The SDSS-DR6 velocity dispersions are not affected by this bias, but they are available only for $85 \%$ of our sample. However, we verified that restricting the analysis to the sample with DR6 velocity dispersions changes the FP coefficients by less than $2 \%$. 
TABLE 1

FP PARAMETERS IN THE $r$ AND K BANDS FOR THE SAMPLE OF 1430 GALA XIES.

\begin{tabular}{c|c|c|c|c|c|c}
\hline \hline RA & DEC & $\log R_{\mathrm{e}, \mathrm{r}}$ & $\langle\mu\rangle_{\mathrm{e}, \mathrm{r}}$ & $\log R_{\mathrm{e}, \mathrm{K}}$ & $\langle\mu\rangle_{\mathrm{e}, \mathrm{K}}$ & $\log \sigma_{0}$ \\
\hline 145.34432 & -0.01692 & 0.860 & 21.602 & 0.527 & 17.046 & 2.282 \\
147.24805 & -0.03572 & 0.834 & 21.346 & 0.680 & 17.437 & 2.228 \\
146.81199 & -0.19005 & 0.459 & 19.968 & 0.349 & 16.319 & 2.217 \\
146.09369 & -0.79309 & 0.985 & 21.521 & 0.475 & 16.216 & 2.259 \\
146.46892 & -0.09284 & 1.622 & 23.236 & 1.090 & 18.009 & 2.317 \\
146.19333 & -0.03887 & 0.625 & 20.555 & 0.445 & 16.439 & 2.226 \\
145.68114 & -0.86722 & 1.001 & 21.576 & 0.721 & 17.350 & 2.196 \\
145.70894 & -0.74768 & 0.134 & 18.936 & 0.088 & 15.653 & 2.112 \\
145.48725 & -0.80693 & 0.965 & 21.993 & 1.065 & 19.167 & 2.180 \\
145.42694 & 0.04954 & 0.470 & 19.852 & 0.018 & 14.473 & 2.367 \\
145.44549 & -0.12268 & 0.381 & 19.576 & 0.166 & 15.351 & 2.353 \\
145.34160 & -0.57727 & 0.087 & 18.124 & -0.152 & 13.574 & 2.355 \\
145.19382 & 0.16887 & 0.192 & 18.862 & 0.137 & 15.669 & 1.930 \\
146.28017 & -0.40695 & 0.033 & 17.760 & -0.129 & 13.996 & 2.288 \\
147.30829 & 0.15116 & 0.830 & 21.046 & 0.454 & 16.295 & 2.287 \\
146.72794 & -0.55688 & 0.262 & 19.223 & 0.178 & 15.409 & 2.316 \\
148.85664 & -0.05916 & 0.978 & 21.643 & 1.160 & 19.055 & 2.226 \\
147.79347 & 0.12326 & 0.614 & 20.337 & 0.227 & 15.566 & 2.187 \\
147.74868 & 0.11584 & 0.480 & 20.362 & 0.125 & 15.682 & 2.233 \\
148.58499 & -0.94207 & 1.180 & 22.575 & 0.703 & 17.637 & 2.109 \\
149.12382 & -0.39828 & 0.365 & 19.682 & -0.037 & 14.726 & 2.324 \\
149.11298 & -0.34883 & 0.685 & 20.638 & 0.593 & 16.954 & 2.270 \\
149.18631 & -0.31181 & 1.291 & 23.217 & 0.823 & 18.064 & 2.101 \\
148.84251 & -0.04411 & 0.469 & 20.031 & 0.387 & 16.276 & 2.202 \\
149.11264 & -0.47563 & 0.572 & 20.065 & 0.471 & 16.069 & 2.398 \\
149.17153 & -0.41298 & 0.359 & 19.539 & 0.266 & 15.894 & 2.260 \\
& & & & & & \\
\hline
\end{tabular}

less affected by outliers (e.g. Jørgensen et al. 1996). We adopted two fitting procedures, minimizing the residuals either in $\log \sigma_{0}$ or in the orthogonal direction to the plane. The first method is virtually independent of selection effects in the plane of photometric parameters, while the orthogonal fit, adopted in most previous works, treats all the variables symmetrically (see La Barbera et al. $2000)$.

The FP coefficients were corrected for selection effects through Monte-Carlo simulations. First, we generated galaxy magnitudes according to the r-band luminosity function of ETGs (Bernardi et al. 2003a). For each magnitude, we derived $\log r_{\mathrm{e}}$ and $\langle\mu\rangle_{\mathrm{e}}$ from the luminosity-size relation of Shen et al. (2003). Values of $\log \sigma_{0}$ were assigned by using the equation of the $\mathrm{FP}$, assuming given values of $a, b$, and $c$, and a given scatter in $\log \sigma_{0}$. All these quantities were chosen to match the observed FP. Notice that when deriving the simulated FP we applied the same cuts in magnitude and $\log \sigma_{0}$ as we did for the real sample. The corrections for selection effects on $a, b$, and $c$, were estimated by not applying the cuts in magnitude and $\log \sigma_{0}$ to the simulated FP. These corrections amount to $+0.01 \%,+8 \%$ and $+5 \%$ for the $\log \sigma_{0}$ fit, and $+35 \%,+7 \%$ and $+17 \%$ for the orthogonal fit. As expected (e.g. La Barbera et al. 2000), the magnitude cut underestimates the coefficient $a$ of the orthogonal fit, while for the $\log \sigma_{0}$ fit the effect is negligible. The above corrections depend mainly on the scatter around the FP, and, because of the very similar dispersions of the r- and K-band FPs (see below), were applied to both the $\mathrm{r}$ - and the K-band coefficients. We notice that the above procedure assumes that our sample of early-type galaxies is magnitude complete. However, because of the matching of the initial volume-complete SDSS catalog with the UKIDSS database, that reduces the sample size from 33628 to 1430 ETGs, the above assumption might not
TABLE 2

FP COEFFICIENTS.

\begin{tabular}{c|c|c|c|c}
\hline \hline & $\mathrm{a}$ & $\mathrm{b}$ & $\mathrm{c}$ & $\mathrm{rms}$ \\
\hline $\log \sigma_{o}$ fit & \multicolumn{3}{|c|}{} \\
$\mathrm{r}-$ band & $1.55 \pm 0.07$ & $0.312 \pm 0.003$ & $-9.1 \pm 0.1$ & 0.081 \\
$\mathrm{~K}$ - band & $1.51 \pm 0.04$ & $0.307 \pm 0.003$ & $-8.6 \pm 0.1$ & 0.073 \\
\hline orthogonal fit & & & & \\
r-band & $1.42 \pm 0.05$ & $0.305 \pm 0.003$ & $-8.8 \pm 0.1$ & 0.064 \\
$\mathrm{~K}$ - band & $1.53 \pm 0.04$ & $0.308 \pm 0.003$ & $-8.6 \pm 0.1$ & 0.062 \\
\hline
\end{tabular}

necessarly hold. To address this point, we retrieved effective parameters and velocity dispersions for the whole sample of 33628 ETGs in the SDSS catalog, and estimated how the r-band FP coefficients change between the whole and the UKIDSS-matched samples. We found the variation to be completely negligible, amounting to $1 \%$ and to $2 \%$ for the values of $a$ and $b$ obtained by the orthogonal fit.

The coefficients of the FP are reported in Tab. 2, In Fig. 11 we compare the edge-on views of the $\mathrm{r}$ - and $\mathrm{K}$ band planes, and in the inset we show the values of $a$ and $b$. The $a$ coefficients differ by only $2 \sigma$ for the orthogonal fit, and they almost coincide for the $\log \sigma_{0}$ fit, which is virtually unaffected by selection effects. The value of $b(\sim 0.308)$ is independent of the waveband, as well as the scatter of the FP, which presents a tiny difference $(<2 \%)$ for the $\log \sigma_{0}$ fit (see also Pahre et al. 1998b). The r-band value of $a$ is fully consistent with that of $a=1.49 \pm 0.05$ found by Bernardi et al. (2003b), while it is larger than that of $a=1.24 \pm 0.07$ found by Jørgensen et al. (1996). For the K-band, the $\log \sigma_{0}$ coefficient is consistent with the value of $a=1.53 \pm 0.08$ found by Pahre et al. (1998b), while it is larger than that of $a=1.38 \pm 0.1$ found by Zibetti et al. (2002). We notice that, because of the large sample size, the accuracy of our 


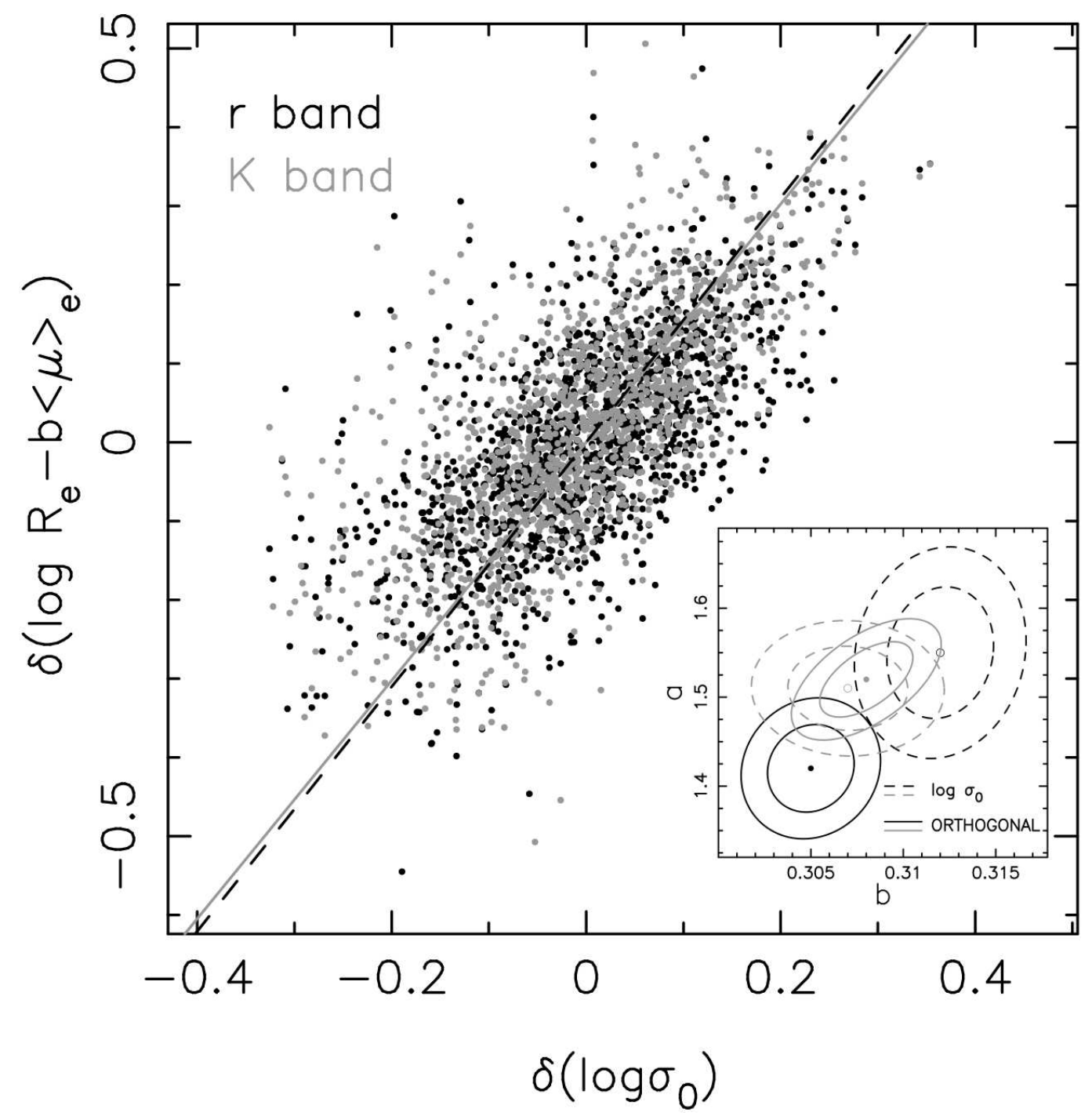

Fig. 1. - Short edge-on view of the $\mathrm{r}$ - and K-band FPs, with the photometric quantity, $\left.\log R_{\mathrm{e}}-b<\mu\right\rangle_{\mathrm{e}}$, being plotted as a function of $\log \sigma_{0}$. Both quantities have been normalized by subtracting their median values, enabling a direct comparison of the $\mathrm{r}$ - and $\mathrm{K}$-band projections. The median $\log \sigma_{0}$ value amounts to $2.215 \mathrm{dex}$. The FPs are represented by two lines, whose slopes are equal to the coefficients $a$ of the $\log \sigma_{0}$ fits. Black and grey colors correspond to the $\mathrm{r}$ and $\mathrm{K}$ bands, respectively. The average value of $b=0.308$ was adopted for both bands. The inset compares the r- and K-band slopes of the FP, with the concentric ellipses denoting 1 and $2 \sigma$ confidence contours. Results of the orthogonal and $\log \sigma_{0}$ fits are represented by solid and dashed ellipses, respectively.

$\mathrm{K}$-band $\mathrm{FP}$ coefficients is significantly higher (by 50\%) than in previous studies. The invariance of the FP with waveband is in agreement with Cappellari et al. (2006), who found for 25 ETGs from the SAURON project the $\mathrm{M} / \mathrm{L}$ versus $\mathrm{L}$ relation to have the same slope in both the I- and K-bands.

\section{CONSTRAINTS ON THE STELLAR POPULATIONS}

The tilt of the FP can be parameterized as a power-law relation between $M / L$ and $M$. We assume that the stellar mass-to-light ratio of galaxies, $M_{*} / L$, is a power-law: $M_{*} / L \propto M^{\beta^{*}}$. This agrees with what was found in previous studies for bright ETGs (see TBB04). The $M / L$ vs. $M$ relation can then be written as $M / L \propto M^{\beta+\beta^{*}}$ (see also Prugniel \& Simien 1996). The quantity $\beta$ is related to the ratio of stellar to total mass as $M_{*} / M \propto M^{-\beta}$, describing the variation of wavelength independent properties with mass (e.g. dark matter content). The quantity $\beta^{*}$ depends on how stellar population parameters vary with mass. Considering only the effects of age and metallicity, for each waveband, we can write:

$$
\frac{\delta\left(\log M_{*} / L\right)}{\delta\left(\log M_{*}\right)}=\frac{\beta^{*}}{1-\beta}=c_{\mathrm{t}} \cdot \frac{\delta(\log t)}{\delta\left(\log M_{*}\right)}+c_{\mathrm{Z}} \cdot \frac{\delta(\log Z)}{\delta\left(\log M_{*}\right)}(1)
$$

where the quantities $\delta(\log t)$ and $\delta(\log Z)$ are the $\log$ arithmic differences of age and metallicity defined between more and less massive galaxies (per decade in stellar mass), while $c_{\mathrm{t}}=\frac{\partial \log M_{*} / L}{\partial \log t}$ and $c_{\mathrm{Z}}=\frac{\partial \log M_{*} / L}{\partial \log Z}$ are the partial derivatives of $\log M_{*} / L$ (in a given band) with respect to $t$ and $Z$. Writing Eq. 1 for $\mathrm{r}$ and $\mathrm{K}$ bands, we obtain two independent constraints on $\delta(\log t)$ and $\delta(\log Z)$. Then, expressing the values of $\beta^{*}$ in the $\mathrm{r}$ and $\mathrm{K}$ bands $\left(\beta_{\mathrm{r}}^{*}\right.$ and $\beta_{\mathrm{K}}^{*}$ ) as a function of the corresponding coefficients of the FP, we can estimate $\delta(\log t)$ and $\delta(\log Z)$. We introduce the parameter $f=\beta_{\mathrm{K}}^{*} /\left(\beta+\beta_{\mathrm{K}}^{*}\right)$ which defines the fraction of the K-band slope of the $M / L$ vs. $M$ relation (i.e. the fraction of the $\mathrm{K}$-band tilt) due to stellar population effects. We note that $f$ can 


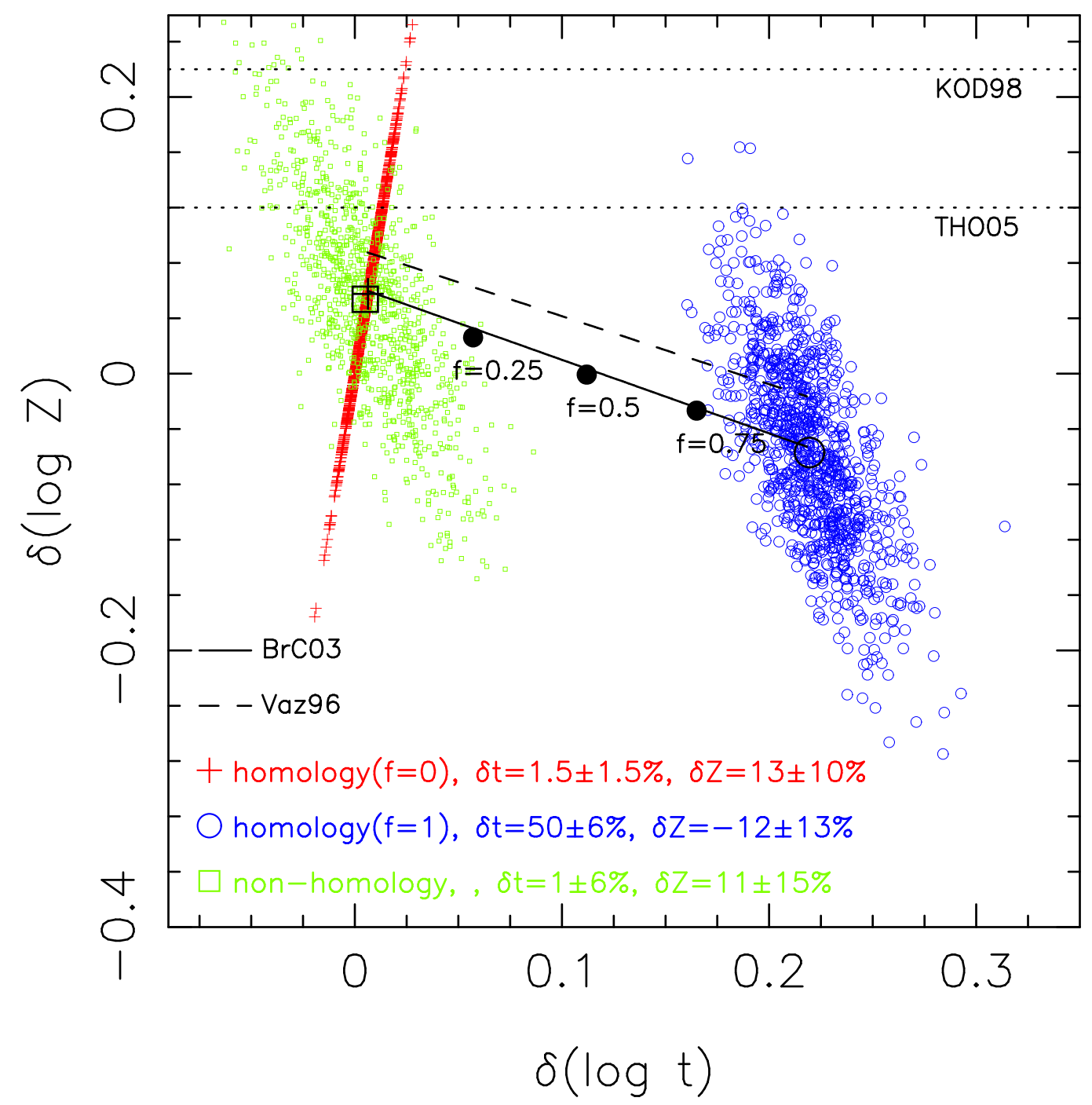

Fig. 2.- Differences of age, $\delta(\log t)$, and metallicty, $\delta(\log Z)$, per decade in mass between more and less massive galaxies, as derived from the FP slopes in the $\mathrm{r}$ and $\mathrm{K}$ bands, by adopting the mean values of $a$ of the orthogonal and $\log \sigma_{0}$ fits (see the text). Red crosses and blue circles correspond to the cases of homology assumption with $f=0$ and $f=1$, respectively, while the green squares correspond to the case where non-homology is taken into account (see Sec. 5). For each symbol type, different points mark the values of $\delta(\log t)$ and $\delta(\log Z)$ obtained by shifting $a_{\mathrm{r}}$ and $a_{\mathrm{K}}$ according to the corresponding uncertainties. For each case, the median values, $\delta \mathrm{t}$ and $\delta \mathrm{Z}$, of $\delta(\log t)$ and $\delta(\log Z)$ are plotted as black symbols and are reported in the lower-left part of the plot. The effect of changing $f$ on the median values of $\delta(\log t)$ and $\delta(\log Z)$ is shown by the black solid and dashed lines, for the BrC03 and the Vaz96 models, respectively. The horizontal dotted lines mark the values of $\delta \mathrm{Z}$ obtained from previous studies of the color-magnitude relation (KOD98) and absorption line indices (THO05).

vary between 0 and 1 . For $f=0$, the K-band tilt is independent of stellar populations $\left(\beta_{\mathrm{K}}^{*}=0\right)$, while for $f=1$ the tilt is entirely explained by stellar population effects $(\beta=0)$. Under the assumption of homology, the slope of the $M / L$ vs. $M$ relation can be written as $(2-a) /(2+a)$. With the above notation, the following relations hold:

$$
\begin{array}{r}
\beta+\beta_{\mathrm{r}}^{*}=(1-f) / f \cdot \beta_{\mathrm{K}}^{*}+\beta_{\mathrm{r}}^{*}=\left(2-a_{\mathrm{r}}\right) /\left(2+a_{\mathrm{r}}\right),(2 a) \\
\beta+\beta_{\mathrm{K}}^{*}=\beta_{\mathrm{K}}^{*} / f=\left(2-a_{\mathrm{K}}\right) /\left(2+a_{\mathrm{K}}\right) .(2 b)
\end{array}
$$

For different $f$ 's and using the values of $a$ in Tab. 2 , we computed $\beta_{\mathrm{r}}^{*}$ and $\beta_{\mathrm{K}}^{*}$ from Eqs. 2, and then, inverting Eq. 1 for both bands, we derived $\delta(\log t)$ and $\delta(\log Z)$. The values of $c_{\mathrm{t}}$ and $c_{\mathrm{Z}}$ were estimated using simple stellar population models, with solar metallicity and an age of 12 Gyr ${ }^{7}$, using both the Bruzual \& Charlot (2003)

\footnotetext{
${ }^{7}$ Computing $c_{\mathrm{t}}$ and $c_{\mathrm{Z}}$ by varying $t$ between 5 and $12 \mathrm{Gyr}$, and $Z$ from $0.5 Z_{\odot}$ to $2.0 Z_{\odot}$, we found that the change of the median
}

(from now BrC03) and the updated Vazdekis (1996) (from now Vaz96) models. We adopted a Scalo IMF and a Salpeter IMF for the BrC03 and Vaz96 models, respectively. Fig. 2 shows $\delta(\log Z)$ versus $\delta(\log t)$ obtained for $f=0$ and $f=1$, as well as the mean values of $\delta(\log t)$ and $\delta(\log Z)$ as a function of $f$. The scatter seen in the plot reflects the uncertainties listed in Tab. 2 for the $a_{\mathrm{r}}$ and $a_{\mathrm{K}}$ coefficients. The figure shows that if the NIR tilt of the FP is not caused by stellar population effects $(f=0)$, more massive galaxies have to be more metal rich than less massive ones $(\delta(\log Z)>0)$, with galaxy ages being remarkably homogeneous $(\delta t / t \sim 1 \%)$. As $f$ increases, we see that $\delta(\log Z)$ decreases, while $\delta(\log t)$ becomes larger. Specifically, for $f \sim 1$, more massive galaxies are much older $(\delta t / t \sim 50 \%)$ and less metal rich than low mass systems.

values of $\delta(\log t)$ and $\delta(\log Z)$ are negligible, amounting to less than 0.01dex and 0.005dex, respectively. 


\section{DISCUSSION}

This work presents the waveband dependence of the FP by comparing the optical and NIR FPs for a large sample of galaxies, with homogeneous measurements of structural parameters and velocity dispersions. This is allowed, for the first time, thanks to the availability of both SDSS r-band photometry and spectroscopy, and UKIDSS K-band photometry for the same sample of ETGs. Such a dataset, together with the use of the same fitting procedure in both bands, makes our study virtually free from any methodological effect on the waveband dependence of the FP. Our analysis shows that the FP does not change significantly from the optical to the NIR, bringing interesting questions about the nature of the sequence of ETGs.

In Sec. 5, we have shown how the r- and K-band FPs constrain the variation of the stellar population properties (age and metallicity) with stellar mass, and how such a constraint is strongly dependent on the fraction, $f$, of the FP tilt resulting from stellar population effects. Previous studies of the color-magnitude (CM) relation and of line-strength indices of ETGs might help us to solve this dependency, by deriving the proper value for $f$. Kodama et al. (1998) showed that the little redshift evolution of the $\mathrm{CM}$ relation implies (i) all the (luminous) ETGs to be equally old and (ii) more massive galaxies to be more metal rich, with a metallicity change of $\delta(\log Z) \sim 0.22$ dex per decade in stellar mass. This finding is qualitatively consistent with that of Thomas et al. (2005), who found absorption-line indices consistent with a metallicity change of $\delta(\log Z) \sim$ 0.12 dex per mass decade. They also found evidence for an age gradient along the sequence of ETGs, with $\delta(\log t)=+0.05 \pm 0.07$ (see their eq.3). Fig. 2 compares these values of $\delta(\log Z)$ with those derived by the optical-NIR FP. For the BrC03 (Vaz96) model, the maximum value of $\delta(\log Z)$, which is consistent with the FP, amounts to $0.06(0.09) \pm 0.04(0.04)$ dex for $f=0$. This value is $4 \sigma(3.7 \sigma)$ lower than that derived by the CM relation, while it is only $1.5 \sigma(0.8 \sigma)$ lower than that found by Thomas et al. (2005). As $f$ increases, the FP requires the value of $\delta(\log Z)$ to decrease, making the above differences even larger. Therefore, reconciling previous estimates of $\delta(\log Z)$ with our results leads to a scenario where $f=0$, which means that the FP tilt is not driven by stellar populations. We have to remark, however, that this interpretation is troublesome, since galaxy colors and line indices are always measured within a given fixed aperture, and the presence of internal population gradients in galaxies can significantly affect the inferred values of $\delta(\log Z)$ and $\delta(\log t)$ (e.g. Scodeggio 2001).

Further constraints come from previous works addressing the origin of the FP tilt itself. Performing a detailed dynamical analysis of twenty-five galaxies, Cappellari et al. (2006) derived $M / L$ ratios consistent with those obtained from the virial theorem in the assumption of homology, concluding that structural and orbital non-homology have a negligible role in the tilt of the FP (see also Zaritsky et al. 2008). In support to this view, they also showed that the variation of the dynamical $M / L$ is correlated with the $\mathrm{H}_{\beta}$ line-strength, thus ascribing most of the tilt to stellar population (age) effects. However, as the authors notice, this result strictly applies to their measurement of the velocity dispersion as the average over an aperture of radius equal to $r_{e}$, a fact that alone might compensate part of the dynamical non-homology. Moreover, most of the galaxies in their sample $(68 \%)$ are fast rotators, while five of them $(20 \%)$ have low velocity dispersion $\left(\sigma=60-85 \mathrm{~km} \mathrm{~s}^{-1}\right)$. As found by Zaritsky et al. (2006) and D'Onofrio et al. (2008), bright and faint spheroids have different FPs, with the tilt becoming larger for galaxies having low velocity dispersion. Hence, the different selection of our sample and that of Cappellari et al. (2006) prevents a straightforward comparison. Bolton et al. (2007) showed that by replacing mean surface brightness with mass density, the FP relation closely approaches the virial theorem expectation, implying that most of the tilt is caused by a variation of dark matter content with galaxy mass. However, the uncertainties on their FP coefficients and the possible biases introduced by the gravitational-lens selection do not definitively exclude the contribution of non-homology to the FP tilt. Our result agrees with Bolton et al. (2007) regarding the minor role played by stellar populations on the tilt. TBB04, agreeing with Busarello et al. (1997) and Graham \& Colless (1997), found that structural and dynamical non-homology can account for more than twothirds of the FP tilt, with the remaining part being explained by stellar population effects. In particular, restricting the analysis to the magnitude-complete subsample, they found that the contribution of stellar populations to the tilt becomes negligible. Notice also that their FPs are derived from different sources, with significantly different coefficients in the optical and NIR wavebands.

To understand how our results may be affected by the assumption of homology, we followed an approach similar to that of TBB04, using spherical, isotropic, non rotating, one-component models of ETGs following the Sersic law (see La Barbera et al. 2005). For each galaxy, we considered the model with the corresponding Sersic index in the r-band, and used that model to correct the central velocity dispersion to the quantity $\sigma_{\mathrm{t}}$ (defined as the square-root of the total specific kinetic energy), the effective radius to the gravitational radius, $r_{\mathrm{g}}$, and to calculate the mean surface brightness within the gravitational radius, $\langle\mu\rangle_{\mathrm{g}}$. Applying the orthogonal fit, we obtain the following equation of the $\mathrm{FP}$ in the $\mathrm{K}$ band: $\log r_{\mathrm{g}} \propto(2.3 \pm 0.2) \log \sigma_{t}+(0.4 \pm 0.02)<\mu>_{\mathrm{g}}$, which is remarkably close to the virial theorem expectation, implying that non-homology may account for the entire tilt. To explore how this result would affect stellar population properties, we normalized the $\mathrm{r}$ - and K-band FP coefficients in such a way to match the virial theorem expectation $(a=2.0$ and $b=0.4)$ in the $\mathrm{K}$ band. Then, we derived the corresponding values of $\delta(\log Z)$ and $\delta(\log t) \quad($ see Sec. 5). As we can see in Fig. 2, accounting for non-homology leads to the same $\delta(\log Z)$ and $\delta(\log t)$ values as those derived under the assumption of homology if the tilt of the FP is not due to stellar populations (i.e. $f=0$ ). As discussed above, this is consistent with what expected from the color-magnitude relation and absorption-line indices of ETGs.

In summary, our analysis suggests a consistent picture where (i) the tilt of the FP does not originate from stellar population effects but is due to other effects, such as non- 
homology; (ii) the SDSS-UKIDSS FPs require more massive galaxies to be mildly more metal rich than less massive systems, and to have extremely synchronized ages, with the age variation per mass decade being smaller than few percent.

We thank R.R. de Carvalho, S.G. Djorgovski, M.Capaccioli, and A.Mercurio for the helpful comments and suggestions. We also acknowledge the referee for his/her helpful suggestions. We also thank A. Vazdekis for providing us with the most recent version of his stellar population code. We have used data from the 2nd data release of the UKIDSS survey (Lawrence et al. (2007)), which is described in detail in Warren et al. (2007). Funding for the SDSS and SDSS-II has been provided by the Alfred P. Sloan Foundation, the Participating Institutions, the National Science Foundation, the U.S. Department of Energy, the National Aeronautics and Space Administration, the Japanese Monbukagakusho, the Max Planck Society, and the Higher Education Funding Council for England.

\section{REFERENCES}

Bernardi, M., et al. 2003a, AJ, 125, 1849

Bernardi, M., et al. 2003b, AJ, 125, 1866

Bernardi, M. 2007, AJ, 133, 1954

Blanton, M.R., Lin, H., Lupton, R.H., et al., 2003, AJ, 125, 2276

Bolton, A.S., et al. 2007, ApJ 665, 105

Bruzual, G., \& Charlot, S. 2003, MNRAS 344, 1000 (BrC03)

Busarello, G., et al. 1997, A\&A 320, 415

Capelato, H.V., de Carvalho, R.R., Carlberg, R.G. 1995, ApJ, 451,525

Cappellari, M., et al. 2006, MNRAS 366, 1126

Ciotti, L., Lanzoni, B., \& Renzini, A. 1996, MNRAS, 282, 1

Djorgovski, S.G., \& Davis, M. 1987, ApJ, 313, 59

Djorgovski, S. 1992, ASPC, 24, 19

Djorgovski, S.G., \& Santiago, B.X. 1993, ESO/EIPD Workshop on structure, dynamics, and chemical evolution of early-type galaxies, Danziger, I.J, Zelinger, W.W., Kjär, eds., (ESO: Garching) p. 59

D'Onofrio, M., et al. 2008, astro-ph/0804.1892

Dressler, A., et al. 1987, ApJ, 313, 42

Gómez, P.L., et al. 2003, ApJ, 584, 210

Graham, A., \& Colless, M. 1997, MNRAS 287, 221

Hjorth, J., \& Madsen, J. 1995, ApJ, 445, 55

Jørgensen, I., Franx, M., \& Kjaergaard, P. 1995, MNRAS, 276, 1341

Jørgensen, I., Franx, M., \& Kjaergaard, P. 1996, MNRAS, 280, 167

Kelson, D.D., Illingworth, G.D., van Dokkum, P.G., \& Franx, M. 2000, ApJ, 531, 137

Kodama, T., et al. 1998, A\&A 334, 99

La Barbera, F., Busarello, G., \& Capaccioli, M. 2000, A\&A, 362, 851
La Barbera, F., Merluzzi, P., Busarello, G., Massarotti, M., \& Mercurio, A. 2004, A\&A, 425, 797

La Barbera, F., et al. 2005, MNRAS 358, 1116

La Barbera, F., et al. 2008, PASP, 120, 681

Lawrence, A., et al. 2007, MNRAS, 379, 1599

Mobasher, B. et al. 1999, MNRAS, 304, 225

Pahre, M.A., de Carvalho, R.R., \& Djorgovski, R.R. 1998a, AJ 116,1606

Pahre, M.A., Djorgovski, S.G., \& de Carvalho, R.R. 1998b, AJ 116,1591

Prugniel, P., \& Simien, F. 1996, A\&A 309, 749

Schlegel, D., Finkbeiner, D.P., \& Davis, M. 1998, ApJ, 500, 525

Scodeggio, MM., et al. 1998, MNRAS, 301, 1001

Scodeggio, M. 2001, AJ, 121, 2413

Shen, S., et al. 2000, 343, 978

Sorrentino, G., Antonuccio-Delogu, \& V., Rifatto, A. 2006, A\&A, 460,673

Thomas, D., et al. 2005, ApJ 621, 673

Trujillo, I., Burkert, A., \& Bell, E. 2004, ApJ 600, 39 (TBB04)

Vazdekis, et al. 1996, ApJS, 106, 307 (Vaz96)

Warren, S.J., et al. 2007, astro-ph/0703037

Zaritsky, D., Gonzalez, A.H., \& Zabludoff, A.I. 2006, ApJ, 638, 725

Zaritsky, D., Zabludoff, A.I., \& Gonzalez, A.H. 2008, astro-ph/0711.2071v1

Zibetti, S., et al. 2002, ApJ 579, 261 